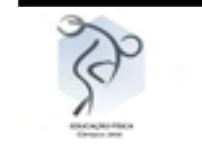

\title{
Treinamento resistido e sistema endócrino: revisão de literatura
}

\author{
Anderson Geremias Macedo ${ }^{1}$ \\ David Michel de Oliveira ${ }^{2}$ \\ Astor Reis Simionato ${ }^{3}$ \\ Anderson Bernardino da Silva ${ }^{4}$ \\ André Tiago Rosito Goes 5 \\ Dalton Müller Pessôa Filho ${ }^{6}$
}

Resumo: O treinamento resistido tem demonstrado eficácia no acréscimo e condicionamento da força muscular, devido às melhorias de aspectos neuromusculares, composição corporal, tolerância às perturbações metabólicas e aspectos endócrinos. Existe uma série de alterações endócrinas causadas pelo exercício resistido. Entretanto, poucos estudos apresentam uma relação conjunta da resposta hormonal frente ao exercício resistido. Deste modo, o objetivo desse estudo foi propor uma descrição, por meio de uma revisão bibliográfica, sobre a resposta da testosterona, GH, IGF-1 e cortisol provocado pelo exercício resistido. O presente estudo tratou-se de uma revisão bibliográfica, realizada na base de dados do Scielo, Google acadêmico, PubMed, abrangendo trabalhos nacionais e internacionais. Como critério de inclusão foi realizada uma análise para verificação dos estudos que apresentavam uma relação direta do título e resumo com a temática do trabalho aqui proposto. A

${ }^{1}$ Pós Doutorando pelo Programa de Pós Graduação em Desenvolvimento Humano e Tecnologias pela Universidade Estadual Paulista Júlio de Mesquita Filho (UNESP), Doutor e Mestre em Ciências Fisiológicas pela Universidade Federal de São Carlos (UFSCAR), graduado em Educação Física pela Faculdade Associadas de Ensino (UNIFAE). E-mail: andersongmacedo@yahoo.com.br

${ }^{2}$ Doutor em Alimentos e Nutrição pela Universidade Paulista Júlio de Mesquita Filho (UNESP), Professor Adjunto III da Universidade Federal de Jatai (UFJ). E-mail: profdoliveira@gmail.com

${ }^{3}$ Mestre em Desenvolvimento Humano e Tecnologias pela Universidade Estadual Paulista Júlio de Mesquita Filho (UNESP), Graduado em Educação Física pela Universidade Estadual Paulista Júlio de Mesquita Filho (UNESP). Docente do Curso de Educação Física da Faculdade Anhanguera de Bauru.

E-mail:astor_ars@hotmail.com

${ }^{4}$ Mestre em Saúde da Comunidade pela Universidade de São Paulo (USP), Graduado em Educação Física pela Universidade Estadual Paulista Júlio de Mesquita Filho (UNESP). Docente do Curso de Educação Física da Faculdade Anhanguera de Bauru. E-mail: silva.edfisica@usp.br

5 Mestre e Doutor em Bioquímica Toxicologia e Farmacológica pela Universidade Federal do Pampa (UNIPAMPA). Graduado em Educação Física pela Universidade Estadual Paulista Júlio de Mesquita Filho (UNESP). Docente do Curso de Educação Física da Faculdade Anhanguera de Bauru.

E-mail: andrerossitogoes@gmail.com

6 Livre-Docente pela Universidade Estadual Paulista Júlio de Mesquita Filho, UNESP, Pós-Doutorado Universidade Politécnica de Madrid, UPM. Mestre e Doutor em Ciências da Motricidade pela Universidade Estadual Paulista Júlio de Mesquita Filho (UNESP). Graduado em Educação Física pela Universidade Estadual Paulista Júlio de Mesquita Filho (UNESP). Professor Associado no curso de Educação Física da Universidade Estadual Paulista Júlio de Mesquita Filho (UNESP). Docente credenciado no Programa de Pós Graduação em Desenvolvimento Humano e Tecnologias pela Universidade Estadual Paulista Júlio de Mesquita Filho (UNESP). E-mail: dalton.pessoa-filho@unesp.br 


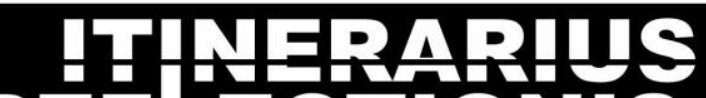 REFLECTIONIS

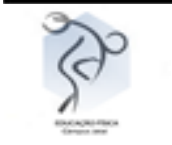

excitação adrenérgica e aumento da concentração de lactato sanguíneo promove liberação de testosterona e GH. O IGF-1 é mediado pelo GH em células hepáticas e, possivelmente, por outras vias não dependente de GH. A secreção de insulina está relacionada a disponibilidade de glicose sanguínea, possuindo papel anabólico no músculo por meio da atividade da mTOR. Já a secreção de cortisol relaciona-se com a redução do volume plasmático e aumentos dos níveis de lactato. Conclui-se que, as diferentes dimensões do TR como intensidade, volume (número de séries e repetições), tipo de ação muscular (concêntrico, excêntrico, isométrico), resposta adrenérgica, volume plasmático e produção de lactato interferem diretamente sobre as concentrações hormonais plasmáticas principalmente em respostas agudas ao exercício.

Palavras - chaves: Treinamento resistido. Sistema endócrino. Exercício físico.

\section{Resistant training and endocrine system: review of literature}

\begin{abstract}
Resistance training has demonstrated efficacy in the addition and conditioning of muscle strength due to improvements in neuromuscular aspects, body composition, tolerance to metabolic disturbances and endocrine aspects. There are a number of endocrine changes caused by resistance exercise. However, few studies present a joint relationship of the hormonal response to resistance exercise. Thus, the objective of this study was to propose a description, through a literature review, of the response of testosterone, GH, IGF-1 and cortisol caused by resistance exercise. The present study was a bibliographical review, carried out in the database of Scielo, Google academic, PubMed, covering national and international works. As inclusion criterion, an analysis was performed to verify the studies that presented a direct relation of the title and abstract with the theme of the work proposed here. Adrenergic excitation and increased blood lactate. concentration promote release of testosterone and GH. IGF-1 is mediated by GH in hepatic cells and possibly by other non-GH dependent pathways. Insulin secretion is related to the availability of blood glucose, having an anabolic role in the muscle through mTOR activity. Cortisol secretion is related to the reduction of plasma volume and increases in lactate levels. It is concluded that the different dimensions of RT as intensity, volume (number of series and repetitions), type of muscular action (concentric, eccentric, isometric), adrenergic response, plasma volume and lactate production interfere directly with plasma hormone concentrations especially in acute responses to exercise.
\end{abstract}

Keywords: Resistance training. Endocrine system. Physical exercise

\section{INTRODUÇÃO}

O treinamento resistido (TR) promove efeitos fisiológicos agudos e adaptações crônicas que são importantes para o aumento da força, potência e hipertrofia muscular (Kraemer, Ratamess, 2005). 
A importância primária para resultado do exercício agudo e subsequente remodelamento muscular é desempenhado pelo sistema endócrino. $\mathrm{O}$ aumento das concentrações hormonais induzidas pelo exercício resistido pode ocorrer pelo aumento de outros hormônios, níveis de lactato, redução do volume plasmático, etc (Kraemer, Ratamess, 2000; Baechle, Earle, 2008). Este aumento da concentração hormonal tem como resposta o crescimento do número e disponibilidade dos receptores ligantes seja na membrana ou no citoplasma, com subsequentes mudanças celulares como aumento da síntese de proteína muscular (Kraemer, Ratamess, 2000; Shoenfeld, 2016).

Alguns hormônios como a testosterona, o hormônio do crescimento (GH), a insulina, o fator de crescimento semelhante a insulina (IGF) e o cortisol estão diretamente envolvidos com o exercício resistido (Kraemer, Ratamess, 2005; Shoenfeld, 2016).

Entretanto, poucos estudos apresentam uma relação conjunta da resposta desses hormônios frente ao exercício resistido. Deste modo, o objetivo desse estudo foi propor uma descrição, por meio de uma revisão bibliográfica, sobre a resposta da testosterona, GH, IGF-1 e cortisol promovida pelo treinamento resistido.

\section{MATERIAIS E MÉTODOS}

Este estudo tem como delineamento a revisão de literatura narrativa. Foram consultados livros e artigos científicos nacionais e internacionais consultados nas bases de dados do Scielo, Google acadêmico e PubMed. Foram utilizados os seguintes descritores testados na Biblioteca Virtual de Saúde (BVS): "exercício resistido", "fisiologia endócrina", "sistema endócrino", "resistance exercise", "endocrine physiology", e "endocrine system". Para o critério de inclusão foi utilizado uma análise verificando os estudos que apresentavam uma relação com a temática do presente estudo. Foram selecionados sua análise foi realizada sobre treinamento resistido com os seguintes hormônios: testosterona, hormônio do crescimento, fator de crescimento semelhante à insulina, insulina e cortisol. A discussão sobre os principais resultados foi exposta nos subtópicos que se seguem. 


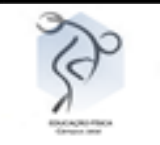

\section{TESTOSTERONA}

Testosterona é um hormônio esteroide derivado do colesterol são sintetizadas nas células de Leydig que se encontram entre os túbulos seminíferos, nos testículos e em pequenas quantidades na adrenal e ovários (Buresh, Berg, Frech, 2009). Os homens apresentam uma quantidade circulante de testosterona 10 vezes maior que as mulheres e provavelmente esta grande diferença hormonal entre os sexos seja em parte responsável pela grande massa muscular observado após puberdade dos homens (Harridge, 2007). A maior concentração da testosterona circulante está ligada a globulina de ligação dos hormônios sexuais (SBHG) cerca de $60 \%$, ou albumina $38 \%$, e na sua forma livre apenas $2 \%$. Somente a testosterona na sua forma livre é biologicamente ativa e disponível para ligar-se aos seus receptores andrógenos (AR) específicos nos tecidos alvos. A interação com seu receptor andrógeno irá formar o complexo hormônio-receptor indo até o núcleo da célula, regulando a transcrição gênica (Kraemer, Ratamess, 2000; Baechle, Earle, 2008)

Tem sido demonstrado que o TR em situação aguda aumenta a secreção de testosterona total somente nos homens (Weiss, Aureton, Thompson, 1983; Chandler, Byrne, Pattersen, 1994). Já a testosterona na sua forma livre parece aumentar tanto nos homens quanto nas mulheres (Baechle, Earle, 2008). A elevação aguda deste hormônio induzidas pelo exercício resistido ocorre pela estimulação adrenérgica, aumento da concentração de lactato e reduções do volume plasmático dos fluidos corporais (Kraemer, Ratamess, 2005). Este hormônio promove o aumento da força por meio da interação com o sistema nervoso central, interagindo-se com receptores neurais e estimulando o neurônio motor (Baechle, Earle, 2008; Kraemer, Ratamess, 2005).

A testosterona tem ações anabólicas, através do aumento do anabolismo muscular e redução do catabolismo (Baechle, Earle, 2008), seus efeitos são amplificados quando combinado com o TR (Kraemer, 2005). Além disso, este hormônio parece auxiliar na regeneração de nervos, aumento do corpo celular e crescimento do diâmetro/comprimento dos dendritos (Brooks et al., 1998). Algumas variáveis do TR parecem ter influência sobre concentrações agudas da testosterona, o trabalho de Buresh, Berg e French (2009) observaram 


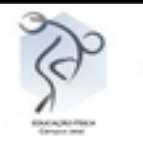

maiores concentrações plasmáticas de testosterona após as primeiras sessões de TR, composto com 1 minuto de intervalo entre as séries quando comparado ao intervalo de 2,5 minutos, isto ocorreu provavelmente pelo aumento das catecolaminas circulantes devido ao menor tempo de repouso entre as séries. Similions e colaboradores (2002) demonstraram voluntários que realizaram 4 séries de 15 repetições nos exercícios supino, puxador costas e agachamento, a concentração plasmática de testosterona foi maior quando comparado aos voluntários que realizaram 2 séries de 15 repetições. O estudo Fahey e colaboradores (1976) verificou que jovens levantadores de peso com mais de 2 anos de experiência apresentaram maiores respostas agudas nas concentrações da testosterona comparado aos com menor experientes. A literatura tem demonstrado que exercícios envolvendo grandes grupos musculares, exercícios com múltiplas séries, intervalos até 1 minuto entre as séries e experiência de dois anos ou mais na prática do exercício resistido são fatores que determinam maior aumento imediato nas concentrações de testosterona (Baechle, Earle, 2008).

Ainda não está claro na literatura as respostas da adaptação crônica da testosterona ao exercício resistido (Baechle, Earle, 2008). Um estudo considerado clássico realizado por Häkkinen e colaboradores (1990) demonstraram que ao longo de dois anos de TR, mesmo em levantadores de peso de elite as concentrações séricas de testosterona em repouso aumentam concomitantemente ao folículo hormônio estimulante e luteinizante, tais substâncias são reguladoras cerebrais superiores da produção de testosterona. Athiainem e colaboradores (2003) observaram significante aumento da testosterona livre e total coletadas em repouso após 7 semanas de TR. Outro estudo do mesmo autor Athiainem e colaboradores (2004) demonstrou que a resposta de testosterona após a sessão de TR em indivíduos atletas foi maior quando comparado aos indivíduos não atletas. Portanto, estudos envolvendo as adaptações dos efeitos crônicos do TR sobre a testosterona ainda não estão bem estabelecidos e parece que o TR aumenta os níveis deste hormônio em repouso e também após a sessão.

O TR também é capaz de modular o receptor andrógeno (Kraemer, Ratamess, 2005). Em ratos, o exercício resistido demonstrou induzir significante aumento na capacidade de ligação do AR no músculo extensor longo dos dedos, mas no músculo sóleo esta capacidade foi reduzida, contudo, demonstra que esta resposta está relacionada ao tipo de 


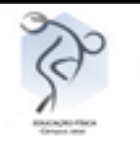

fibra muscular recrutada pelo modelo de treinamento (Dechenes e colaboradores, 1994). A estimulação elétrica no músculo gastrocnêmio de ratos aumentou em $25 \%$ o número de AR durante 3 dias, este aumento foi concomitante com o aumento da massa muscular (Ionue e colaboradores, 1993). Bamman e colaboradores (2001) observaram que 48 horas após única sessão de agachamento, houve aumento do mRNA do AR no músculo vasto lateral. Este efeito do exercício sobre AR parece estar associado com o volume de treino. Visto que Ratamess e colaboradores (2005) compararam dois protocolos de agachamento (1 série vs 6 séries de 10 repetições máximas), e nas modificações do AR foram encontradas exclusivamente com o protocolo com 6 séries de 10 repetições. Portanto o TR também promove alterações nos AR.

\section{HORMÔNIO DO CRESCIMENTO (GH)}

O hormônio do crescimento (GH) tem características anabólicas e origem polipeptídica e secretado pela glândula da pituitária, é secretado de maneira pulsátil com picos de liberação durante o sono (Kraemer, Ratamess, 2005 Baechle, Earle, 2008; Shoenfeld, 2016).

O GH tem circulação livre e sua interação com receptor ocorre na membrana para sinalização intracelular, mecanismo denominado como segundo mensageiro (Baechle, Earle, 2008; Shoenfeld, 2016).

As principais funções do GH estão relacionadas ao metabolismo de substratos com baixa utilização de glicose e alta lipólise, redução da síntese de glicogênio, aumento do transporte de aminoácidos e síntese de proteínas, atua também como contrarregulador de hormônios do catabolismo (Shoenfeld, 2016)

O TR em situação aguda estimula a secreção do GH, este aumento parece ocorrer devido a excitação adrenérgica e principalmente pelo aumento da concentração de lactato plasmático (Kraemer, Ratamess, 2005, Baechle, Earle, 2008). 
A elevação aguda de suas concentrações parece não aumentar o processo de síntese de proteína, porém demonstra promover o aumento do transporte de aminoácidos para célula muscular (Shoenfeld, 2016).

Importantes fatores como volume do treinamento, intervalo entre as séries, tipos de exercícios podem interferir diretamente sobre as concentrações plasmáticas do GH. Similions et al. (2002) observaram que indivíduos que realizaram uma sessão de exercício com supino, puxador costas e agachamento com 4 séries de 15 repetições (leve) a concentração plasmática de GH foi maior comparado aos indivíduos que realizaram 4 séries com 10 (moderada) ou 5 (vigorosa) repetições. Além disso, neste mesmo estudo os indivíduos que realizaram 4 séries de 15 repetições apresentaram maior concentração do GH comparados aos indivíduos que realizaram somente 2 séries de 15 repetições, mostrando que intensidades mais moderada e maior volume do TR promove maiores aumentos da concentração de GH durante o exercício resistido. O estudo de Martin e colaboradores. (2007) mostraram mulheres jovens que treinaram com intervalos entre as séries de 30, 60 segundos, a concentração de GH foi maior comparado as voluntárias que utilizaram os intervalos de 120 segundos devido esta resposta parece ter sido mediada pelas maiores concentrações plasmática de lactato nos grupos de 30 e 60 segundos.

Por fim indivíduos jovens apresentaram maior secreção de GH comparado aos idosos após a sessão de TR isto se deve pela redução da secreção deste hormônio a partir dos 30 anos (Kraemer e colaboradores, 1999). De um modo geral, fatores ligados ao exercício resistido como intensidade, volume, intervalos até 1 minuto entre as séries, tipo de contração especifica e idade podem influenciar as respostas agudas deste tipo de exercício sobre o GH (Baechle, Earle, 2008).

O TR parece não afetar os níveis de GH em repouso. Não tem sido demonstrada alterações nas concentrações do GH tanto em homens quanto em mulheres de várias idades (Athiainen e colaboradores, 2003; Kraemer, Ratamess, 2005). A literatura também demonstra níveis semelhantes das concentrações de GH em repouso entre indivíduos poucos treinados e atletas de levantamento de peso olímpico ou de força (Hakkinen e colaboradores, 1998; Athiainen e colaboradores, 2003). 


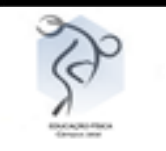

\section{FATOR DE CRESCIMENTO SEMELHANTE À INSULINA 1 (IGF-1)}

Muitos efeitos do GH são mediados por meio de pequenos polipeptídios chamados de fator de crescimento semelhante a insulina-1 (IGF-1), este polipeptídio apresenta estrutura semelhante a insulina (Baechle, Earle, 2008; Shoenfeld, 2016). Este hormônio é secretado pelo fígado após a estimulação do GH nas células hepáticas (Baechle, Earle, 2008). A sinalização deste hormônio ocorre via segundo mensageiro e as cascatas de sinalização intracelular tem efeito anabólico e anticatabólico no tecido estriado, diante disso ocorre crescimento muscular (Kraemer, Ratamess, 2005; Shoenfeld, 2016).

As respostas do IGF-1 ao TR ainda não estão totalmente esclarecidos. Muitos estudos demonstraram mudanças deste hormônio durante e imediatamente após o exercício resistido (Kraemer, Ratamess, 2005; Shoenfeld, 2016). Este hormônio tem sido um dos mais estudados frente ao TR devido a sua função anabólica. Mas as mudanças induzidas agudamente pelo exercício resistido não estão claras, visto que são necessárias de 8-16 horas para que haja a produção do IGF-1 pelo tecido hepático após a estimulação realizada pelo GH. Entretanto, é possível que o IGF possa ser liberado de células não hepáticas sem a mediação do GH (Kraemer, Ratamess, 2005). O IGF-1 apresenta diferentes isoformas e parece que sua produção pode ser endócrina e autócrina, duas dessas isoformas foram identificadas na musculatura esquelética. A isoforma é similar ao IGF-1 hepático, chamada IGF-1Ea e a segunda isoforma especifica do musculo é o fator de crescimento mecânico (MGF) (Kraemer, Ratamess, 2005). Parece que a isoforma IGF-1Ea é estimulada pela sobrecarga e subsequente dano muscular. Bamman e colaboradores (2001) relataram significantes elevações do IGF-1Ea mRNA imediatamente após a sessão de TR, particularmente durante a contração muscular excêntrica. Hammed e colaboradores (2003) examinaram o músculo após 2 horas e 30 minutos do exercício resistido em homens jovens e houve um aumento significativo do MGF mRNA, porém nos idosos não houve alterações. $\mathrm{O}$ trabalho de Brahn e colaboradores (1997) mostraram que a concentração arterial do IGF-1 


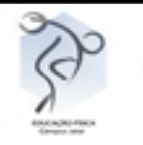

permaneceu constante durante o exercício resistido intenso. Entretanto, a concentração venosa do IGF-1 aumentou, isto sugere que esta elevação da circulação pode ser considerada pela grade liberação mediada pelo músculo devido a ruptura celular e grande fluxo sanguíneo. Aparentemente o aumento do IGF-1 induzido de forma aguda não parece ser via endócrina, porém ainda são escassos na literatura estudos sobre o tema.

Os níveis basais das concentrações ainda do IGF-1 induzido como resposta adaptativa ao TR requer maiores investigações. O trabalho de Borst e colaboradores (2001) reportaram significantes elevações do soro de IGF-1 basal somente a partir da $13^{\mathrm{a}}$ semana de um programa de treinamento resistido de 25 semanas. Esta elevação encontrada foi similar entre os grupos que realizaram série única e múltiplas séries apesar do significante aumento de força observado no grupo de múltiplas séries. Resultados similares foram encontrados por Marx e colaboradores (2001) observaram significantes elevações das concentrações do IGF-1 de repouso em mulheres sedentárias após 6 meses de TR. Assim como o GH, a adaptação ao treinamento resistido e IGF-1 são refletidas provavelmente em variedades de mecanismos como transporte e interação com receptor (Baechle, Earle, 2008). Além disso, investigações futuras são necessárias para determinar adaptações agudas e crônicas na produção do IGF-1Ea e MGF pelo musculo esquelético.

\section{INSULINA}

A insulina é um hormônio polipeptídio secretado pelas células betas do pâncreas. Em indivíduos saudáveis a insulina regula o metabolismo de glicose através da facilitação dos estoques de glicose no músculo e no fígado (Baechele, Earle, 2008; Shoenfeld, 2016). Dentre outros papéis secundários, a insulina está envolvida no anabolismo muscular, estimulando as fases de iniciação e alongamento da tradução proteica pela regulação de vários elFs e eEFs (Shoenfeld, 2016). Este hormônio também pode exercer efeito anabólico por meio da ativação de uma proteína universalmente abreviada como mTOR. A mTOR desempenha um papel crítico na regulação do crescimento celular e monitoramento nutricional celular, nos níveis energéticos e de oxigênio (Macedo e colaboradores, 2014; Shoenfeld, 2016). Apesar de 


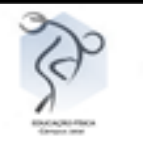

suas propriedades anabólicas (Kraemer, Ratamess, 2005), a insulina induzida pelo TR parece reduzir o catabolismo de proteínas contribuindo para a hipertrofia muscular (Heslin e colaboradores, 1992).

\section{CORTISOL}

Os glicocorticóides são liberados pela região do córtex adrenal em resposta ao estresse ou exercício físico. Aproximadamente 95\% das atividades dos glicocorticóides ocorre por meio do cortisol, este hormônio tem funções catabólicas especialmente em fibras musculares do tipo II (Kraemer, Ratamess, 2005; Macedo e colaboradores, 2014).

Somente $10 \%$ do cortisol circulante encontra-se na sua forma livre, $15 \%$ ligado a albumina, e 75\% interage com a globulina ligadora de corticosteróide. Em tecidos periféricos, o cortisol estimula a lipólise nas células adiposas, aumenta da degradação e decréscimo da síntese de proteínas das células musculares resultando em grande liberação de lípidos e aminoácidos na circulação sanguínea (Baechle, Earle, 2008; Macedo e colaboradores, 2014; Macedo e colaboradores, 2016).

Alguns estudos mostraram significativo aumento do cortisol e do hormônio adrenocorticotrópico (ACTH), hormônio da pituitária que estimula a liberação do cortisol no córtex adrenal durante e agudamente após TR (Häkkinen e colaboradores, 1988; Kraemer e colaboradores, 1999). Os mecanismos responsáveis pelo aumento da secreção do cortisol estão relacionados com a redução do volume plasmático e aumentos dos níveis de lactato (Baechle, Earle, 2008). Alguns fatores do treinamento podem interferir nas respostas agudas da concentração de cortisol pelo TR. Similions e colaboradores (2002) demonstraram que grupos que realizaram única sessão de exercício com supino, puxador costas e agachamento, com 4 séries de 15 repetições (leve) e 4 séries de 10 repetições (moderada) tiveram a concentração do cortisol maior comparado ao grupo que realizou 4 séries de 5 repetições (vigorosa). Os mesmos autores verificaram que os níveis de cortisol foram maiores no protocolo de 4 séries comparado a 2 séries com 15 repetições. Buresh, Berg e French (2009) observaram que concentração de cortisol nas primeiras sessões de TR foi maior com 1 minuto 


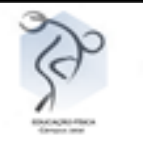

de intervalo entre as séries comparado a 2,5 minutos. Desta maneira, intensidade leve a moderada, treinos volumosos, intervalos até 1 minuto entre as séries podem ser fatores que colaboram para maiores aumentos nas concentrações agudas de cortisol induzida pelo TR (Baechele; Earle, 2008).

Cronicamente o TR parece não produz padrões significativos consistentes nas concentrações de cortisol em repouso (Kraemer, Ratamess, 2005). Athiainem e colaboradores (2004) não encontraram diferenças nas concentrações de cortisol em repouso e após a sessão de exercício resistido em indivíduos atletas e indivíduos não atletas. Desta maneira, a adaptação das concentrações de cortisol frente ao TR parecem não serem consistentes. A razão testosterona/cortisol ou testosterona isolada e/ou testosterona livre/cortisol tem sido sugerido como indicador de estado anabólico/catabólico da musculatura esquelética durante o TR, sendo o aumento desta razão um indicador anabólico e a redução um indicador catabólico. Isto pode ocorrer pelo aumento da testosterona, redução do cortisol ou ambos (Baechele, Earle, 2008). Alguns estudos têm mostrado melhoras nesta razão durante o treinamento de força ou potência (Alen e colaboradores, 1998; Kraemer, Ratamess, 2005). Diante da melhora da razão testosterona/cortisol induzida pelo treinamento resistido é possível que com o aumento através do aumento da testosterona e a redução do cortisol contribua para a hipertrofia do hormônio anabólico contribua para a hipertrofia muscular.

\section{CONCLUSÕES}

A excitação adrenérgica e aumento da concentração de lactato sanguíneo promovem aumento na liberação da testosterona (Kraemer, Ratamess, 2005) e GH (Kraemer, Ratamess, 2005, Baechle, Earle, 2008). O IGF-1 é mediado pelo GH em células hepáticas (Baechle, Earle, 2008) e, possivelmente, por outras vias não dependentes de GH (Kraemer, Ratamess, 2005). A secreção de insulina está relacionada a disponibilidade de glicose sanguínea, possuindo papel anabólico no músculo (Kraemer, Ratamess, 2005) por meio da atividade a mTOR (Macedo e colaboradores, 2014; Shoenfeld, 2016). Por fim, a secreção de cortisol relaciona-se com a redução do volume plasmático e aumentos dos níveis de lactato (Baechle, Earle, 2008). 
Em relação às respostas hormonais agudas ao $\mathrm{TR}$, intervalos de até 1 minuto quando comparados a 2,5 minutos apresentaram maiores concentrações plasmáticas de testosterona e cortisol após as primeiras sessões de TR (Buresh, Berg, French, 2009). O mesmo ocorreu com o $\mathrm{GH}$ quando comparado séries até 1 min com 2 min (Martin e colaboradores, 2007).

Se tratando do volume de TR, mais precisamente do número de séries, também parece influenciar na resposta hormonal. Comparando 2 com 4 séries com o mesmo número de repetições, houve maior concentração sérica de testosterona, GH e cortisol após a realização de 4 séries (Similions e colaboradores, 2002). Sobre o aumento de mRNA do receptor andrógeno, parece que o volume em números de séries também sofre influência, comparando protocolos de 1 série com 6 séries (10 repetições máximas), apenas com maiores séries obtive-se aumento de mRNA do receptor andrógeno (Ratamess e colaboradores, 2005), aumentando a sensibilidade e sinalização da testosterona.

Ainda relacionado ao volume de TR à resposta hormonal, porém em relação de repetições, quando comparado 4 séries em diferentes números de repetições intensidades (15, 10 e 5 repetições), houve maior secreção de GH nas séries mais curtas e intensas (Similions e colaboradores, 2002). O mesmo estudo analisou a resposta do cortisol para os mesmos protocolos, e verificaram que este hormônio estava mais elevado nas séries longas (15 e 10 repetições) que na série curta e intensa (5 repetições).

Em se tratando do tipo de contração muscular, as excêntricas promoveram elevações significantes no mRNA da isoforma IGF-1Ea (Bamman e colaboradores, 2001), imediatamente após o TR.

Entretanto, em relação às respostas crônica ao TR, sobre os níveis de testosterona, em 7 dias de TR parece aumentar significativamente os níveis séricos de repouso (Athiainem e colaboradores, 2003), porém mais estudos são necessários para reforçar esses resultados. Em relação ao $\mathrm{GH}$, os estudos não apresentam alterações nas concentrações séricas de homens e mulheres (Athiainen e colaboradores, 2003; Kraemer, Ratamess, 2005) e treinados e não treinados (Hakkinen e colaboradores, 1998; Athiainen e colaboradores, 2003). Em relação as concentrações séricas de IGF, houve aumento significativo do soro de IGF-1 basal após 3 


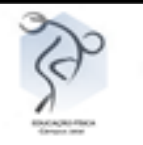

meses de treinamento (BORST et al., 2001) e em mulheres sedentárias após 6 meses (Marx e colaboradores, 2001). E, em relação ao cortisol, cronicamente o TR parece não produz padrões significativos consistentes nas concentrações de repouso (Athiainem e colaboradores, 2004; Kraemer, Ratamess, 2005).

Conclui-se que, as diferentes dimensões do TR como intensidade, volume (número de séries e repetições), tipo de ação muscular (concêntrico, excêntrico, isométrico), resposta adrenérgica, volume plasmático e produção de lactato interferem diretamente sobre as concentrações hormonais plasmáticas principalmente em respostas agudas ao exercício.

\section{REFERÊNCIAS}

AHTIAIMEN, J.P; PAKARINEN, A.; ALEN, M et al. Muscule hypertrophy, hormonal adaptations and strength development during strength training in strength-trainde an untrained men. Eur J Appl Physiol, Vol. 89, Num 6. 2003. p.55-563.

AHTIAINEN, J.P.; PAKARINEN, A.; KRAEMER, W.J.; HAKKINEN, K. Acute hormonal responses to heavy resistance exercise in strength athletes versus nonathletes. Can $J$ Appl Physiol Vol. 29. Num. 5. 2004. p.527-43.

ALEN, M.; PAKARIN, A.; HAKKINEN, K. et al. Responses of serum androgenicanabolic an catabolic hormones prolonged strength training. Int J Sports, Vol.9, Num 3. 1998. p. 229-233.

BAECHLE, T.R.; EARLE, R.W. Essentials of strength training and conditioning. 3 ed. Champaign: Human Kinetics, 2008. p 752.

BAMMMAN, M.M.; SHIPP, J.R.; JIANG, J. et al. Mechanical load increase muscle IGF-1 aand androgen receptor mRNA concentrations in humans. Am $J$ Physiol Endocrinol Metab. Vol. 280. Num.3 2001. p. 383-90.

BORST, S.E.; DE HOYOS, D.V.; GAZARELLA, L. et al. Effects of resistance training on insulin-like growth factor-I and IGF binding protein. Sci Sports Exerc, Vol. 33. Num 4. 2001. p.648-53.

BRAHN, H. PIEHL-AULIN, K., SALTIN, B. et al. Net fluxes over working trigh of hormones, growth the factor and biomarkers of bone metabolism during short lasting dynamic exercise. Calcif tissue Int, Vol. 60. Num. 2. 1997. p. 175-80. 


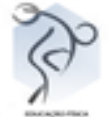

BROOKS, B.P.; MERRY, D.E.; PAULSON, H.L et al. A cell culture model for androgen effects motor neurons. J Neurochem, Vol.70. Num 3. 1998. p. 1054-1060,

BURESH, R.; BERG, K.; FRENCH, J. The effect of resistive exercise rest interval on hormonal response, strength, and hypertrophy with training. J Strength Cond Res. Vol. 23. Num.1. 2009. p.62-71.

CHANDLER, R.M.; BYRNE, H.K. PATTERSEN, J.G. Dietary supplements affect the anabolic hormones after weight-training exercise. J appl Pysiol. Vol. 78. Num 2. 1975. p. 839-845.

DESCHENE, M.R.; MARESH, C.M. AMSTRONG, L.E. et al. Endurance and resistance exercise induced fiber type specific in androgen binding receptor. J Steroid Biochem Mol Biol. Vol. 52. Num 3-4. Ano 1994. p. 175-179.

FAHEY, T.D.; ROLPH, R.; MOUNGMEE, P et al. Serum testosterone, body composition, and strength of young adults. Med Sci sports Exerc. Vol. 8. Num 1. 1976. p. 13-34.

HAKKINEN, K.; PAKARAIMEN, A.; KYROLAIMEN, S. et al. Neuromuscular adapatation and serum hormones in fameless during prolonged power training. Int $\mathrm{J}$ Sports Med. Vol.11. Num 2. 1990. 91-98.

HAKKINEN, K.; PAKARIMEN, A.; ALEN, M. et al. Neuromuscular and hormonal response in elite athletes to two successive strength training sessions in one day. Eur $\mathrm{J}$ Appl Physiol Vol. 57. Num 2. 1988. p. 133-139.

HAKKINEN, K.; PAKARINEN, A.; ALEN, M. et al. Neuromuscular and hormonal adaptation in athletes to strength training in two year. $\mathrm{J}$ Appl Physiol. Vol. 65. Num 6. 1998. p. 2406-12,

HAMMED, M.; ORREL, R.W.; COBOLD, G. et al. Expression of IGF-1 splice variants in young and old human skeletal muscle after high resistance exercise. J Phsiol. Vol. 547. Num 1. 2002. p. 247-54.

HARRIDGE, S.D. Plasticity of human skeletal muscle: Gene expression to in vivo function. Exp Physiol, Vol. 92. Num 5. 2007. p. 783-792.

INOUE, K.; YAMASAKI, S. FUSHIKI, T. et al. Rapid increase in the number of androgen receptors following electrical stimulation of the rat muscle. Eur. J. Appl Physiol. Vol. 66. Num. 1993. p.134-140.

KRAEMER W.J.; FLECK, S.J.; MARESH, C.M. et al. Acute hormonal response to single bout of heavy resitance exercise in trained power lifters and untrained men. Can J Appl Physiol, Vol. 24. Num 6. 1999. p. 524-37. 


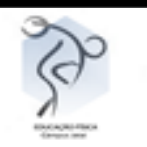

KRAEMER, W.J.; DUDLEY, G.A.; TESCH, P.A et al. The influence of muscle action on the acute growth hormone response to resistance exercise and short-term detraining. Growth Horm IGF Res. Vol.11. Num 2. 2001. p. 75-83.

KRAEMER, W.J.; HAKKINEN, K.; NEWTON, R.U et al. Effect of heavy resistance training on hormonal patterns in younger vs. older men. J Appl Physio. Vol.87. Num. 3. 1999. p.982-92.

KRAEMER, W.J.; RATAMESS, N.A. Hormonal responses and adaptations to resistance exercise and training. Sports Med. Vol. 35. Num.4. 2005. p.339-361.

KRAEMER, W.J.; RATAMESS, N.A. Physiology of resistance training: current issue. Orthop Physn Ther Clin North Am Exercise Technol, Vol. 9. Num 4. 2000. p. 467-513.

MACEDO, A.G.; KRUG, A.L.O, SOUZA, L.M.; MARTUSCELLI, A.M.; CONSTANTINO, P.B.; ZAGO, A.S. et al. Time-course changes catabolic proteins following muscle atrophy induced by dexamethasone. Steroid. Vol.107. 2015. p.30-36.

MACEDO, A.G.; KRUG, A.L.O., HERRERA, N.A., ZAGO, A.S.; RUSH, J.W.E. et al. Lowintensity resistance training attenuates dexamethasone-induced atrophy in the flexor halluces longus muscle. J Steroid Biochem Mol Biol. Vol.143. Num 143. 2014. p.357-364,

MARX, J.O.; RATANESS, N.A.; NINDL, B.C. et al. Low-volume circuit training versus hig-volume periodized resistance training. Vol.33. Num 4. 2001. p. 635-43.

RATAMES, N.A.; KRAEMER, W.J.; VOLEK, J.S. et al. Effect of heavy resistance volume on past-exercise androgen receptor content in resistance-trained men. J Steroid Biochem Molec Biol. Vol. 93. Num 1. 2005. p. 35-42,

SHOENFELD, B. Science and development of muscle hypertrofphy. 1 ed. Champaign: Human Kinetics. 2016. p 224.

SIMILIOS, I.; PILIANIDIS, T.; KARAMOUZI, M.;TOKMAKIDIS, S.P. Hormonal response after various resistance exercise protocols. Med Sci Sports Exerc, Vol.35. Num. 4. 2003. p. 644-54.

WEISS, L.W.; AURETON, K.J. THOMPSON, F.N. Comparison of serum testosterone and androstenedione responses to weight lifting in men and women. Eur J Pahysiol. Vol. 50. Num 3. 1983. p. 413-419. 